\title{
Glucocorticosteroid receptors in ovarian carcinomas
}

\author{
JOACHIM WOENCKHAUS ${ }^{1}$, FOLKER E. FRANKE ${ }^{1}$, ANDREAS HACKETHAL ${ }^{2}$, \\ RICHARD VON GEORGI ${ }^{3}$ and KARSTEN MÜNSTEDT ${ }^{2}$ \\ ${ }^{1}$ Institute of Pathology, ${ }^{2}$ Department of Obstetrics and Gynecology, Clinic of the Universities Giessen and Marburg; \\ ${ }^{3}$ Institute of Medical Psychology and Sociology of the Justus-Liebig University, Giessen, Germany
}

Received November 8, 2005; Accepted December 27, 2005

\begin{abstract}
The use of glucocorticoids (GCs) in oncology, including in the treatment of ovarian carcinomas, is controversial. In vitro experiments suggest that GCs negatively influence the response to chemotherapy, but the few available clinical data show only benefits. Glucocorticoid action is mediated via glucocorticoid receptors (GRs). This study aims to define any clinical implications of GR expression in ovarian cancer to further the debate. Archived tissue samples from patients with histologically confirmed ovarian cancer were analyzed for GR expression and evaluated by immunohistochemistry and immunoreactive score. The results were related to the patients' overall survival. Kaplan-Meier survival and residual survival analyses gave no evidence that GR expression had any prognostic value in the 85 cases studied. No evidence of poorer survival was found in a small subset of GR-positive patients who received GC treatment. Glucocorticoid receptor expression had no prognostic impact in our study. However, GC (cortisol) is being produced continuously by the body, which may have stimulated GR-positive ovarian cancer cells. Our finding does not exclude the possibility that long-term GC treatment has adverse effects, and it should also be emphasized that treatment duration, dosage and dosing regimens, as well as the choice of an appropriate GC and the mode of application, determine the risks and benefits. Our study showed no evidence against using GC for antiemetic prophylaxis in ovarian carcinomas.
\end{abstract}

\section{Introduction}

Glucocorticoids (GCs) are widely used in oncology and have clear and proven benefits in several indications, such as antiemesis. However, the mechanism of action and possible risks associated with GCs are not fully understood, and concerns about the safety of GCs have been expressed. In the

Correspondence to: Dr Karsten Münstedt, Klinikstrasse 32, D 35385 Giessen, Germany

E-mail: karsten.muenstedt@gyn.med.uni-giessen.de

Key words: chemotherapy, glucocorticoid receptor, corticosteroids, ovarian cancer, prognosis late 1950 s and early 1960 s, autopsy studies reported that patients treated with GCs showed a significant increase in the degree of metastatic spread to various organs, including the lung, liver, heart, opposite breast, brain, spleen and submucosa of the gastroduodenum (1-3). Evidence from some in vitro studies suggests that GCs may have an adverse effect on outcome in several cancers, including ovarian cancer (4-7). However, other investigators have reported favorable effects of GCs in vitro (8-10). The fact that in vitro studies may not reflect the clinical situation adds to the continuing uncertainty.

Glucocorticoids play an important part in the treatment of leukemia $(11,12)$. The first clinical data from a retrospective study have shown that GC treatment was not associated with any disadvantage in terms of survival. On the contrary, patients given GCs had significantly higher leukocyte values in the days immediately after chemotherapy, higher nadir leukocyte values, and higher counts before subsequent courses of chemotherapy than patients who did not receive this treatment (13). Furthermore, the introduction of taxanes, which have reactions that are averted by GC treatment, did not lead to a worsened outcome in ovarian cancer $(14,15)$. Unfortunately, the original published report of the International Collaborative Ovarian Neoplasm (ICON3) trial, a study comparing treatment with taxanes and platinum derivatives with platinum alone or with taxanes and cyclophosphamide, does not give details of any GC-based antiemetic medication.

Thus, the use of GC in oncology is still beset by speculation and the lack of pathoclinical data. The action of GCs is mediated by receptors. The present study aims to define the prognostic role of glucocorticoid receptors (GRs) expressed in ovarian cancer as a way of shedding further light on the clinical use of GCs in treating patients with this type of cancer.

\section{Patients and methods}

Patients. We re-evaluated the clinical records of all patients with ovarian carcinoma referred to, treated, and followed-up at the Department of Obstetrics and Gynecology of the Justus-Liebig-University, Giessen, between 1982 and 1996, and recorded the details of prognostic factors (patient age, tumor stage, presence of ascites and tumor size), survival status and, where relevant, the cause of death (classified as cancer-associated, cancer-independent, and unclear). The availability of complete clinical data and tumor tissue from 
initial tumor surgery was the only selection criterion for inclusion in the study.

Tissues and pathology. Specimens of all primary tumors were routinely fixed in formalin and embedded in paraffin wax, and 2-to 3- $\mu \mathrm{m}$ thick tissue sections were cut. Histological diagnoses were performed and re-evaluated on standardized hematoxylin-and-eosin-stained sections following the suggestions of Russell and Farnsworth (16). Tumor grading was performed according to the recommendations of Baak et al (17). Representative tumor specimens from each case were selected for immunohistochemistry.

Immunohistochemistry. Commercially available mouse monoclonal GR antibody (BuGR2) (ab 2768) raised against partially purified rat GR was obtained from Abcam Ltd. (Cambridge, UK). Slides were dewaxed in xylene, rehydrated through a graded ethanol series, and washed in Tris-buffered saline. Endogenous peroxidase was blocked by placing slides in $3 \%$ hydrogen peroxide for $10 \mathrm{~min}$. Antigen retrieval was performed in $0.01 \mathrm{~mol} / 1$ sodium citrate buffer, $\mathrm{pH}$ 6.0, for $25 \mathrm{~min}$ at $98^{\circ} \mathrm{C}$ in a microwave oven. Primary antibodies were diluted according to the manufacturer's instructions in Dako antibody dilutent $(1: 200)$ and incubated overnight at $4^{\circ} \mathrm{C}$ (Dako, Hamburg, Germany). Reactions were detected using the DAB detection kit (Dako). Negative controls for BuGR2 were treated identically, without use of the primary antibody. Immunohistochemical results were evaluated by two different pathologists.

In order to allow comparisons with other steroid receptors and exclude cross-antigenicity, data on estrogen receptor (estrogen receptor monoclonal antibody clone 1D5; Dako) and progesterone receptor (polyclonal rabbit anti-human progesterone receptor antibody; Dako) expression levels from an earlier study were added to the data set (18).

Evaluation and statistics. Immunohistochemistry was semiquantitatively evaluated using the immunoreactive score (IRS), which has been described previously $(18,19)$. IRS is the product of the intensity of immunostaining (none, 0 ; weak, 1; moderate, 2; and strong, 3) and the percentage of positive tumor cells (none, $0 ;<10 \%, 1 ; 10-50 \%, 2 ; 51-80 \%, 3$; and $>80 \%, 4)$. For the statistical analyses of GR expression, an IRS value of 0 was scored as 0 (no expression), values of 1-3 were scored as 1 (poor expression), 4-8 as 2 (moderate expression), and 9-12 as 3 (strong expression). Dichotomized data of GRs (no and poor expression $=0$ versus moderate and strong expression $=1$ ) were used for the analyses of GRs and clinical use of GC. Data management and statistics (bivariate correlation and Kaplan-Meier survival analysis and residual analysis) were performed using the SPSS software for Windows $^{\mathrm{TM}}$, release 11.0 . A probability of error value $<5 \%$ was regarded as significant.

\section{Results}

Patients. The records of 85 patients were evaluated. Tumorassociated death was recorded in 47 patients, who had a mean survival time of 2.2 years (median, 1.4; SD, 2.1). The mean age of patients at diagnosis was 57.7 years (median,

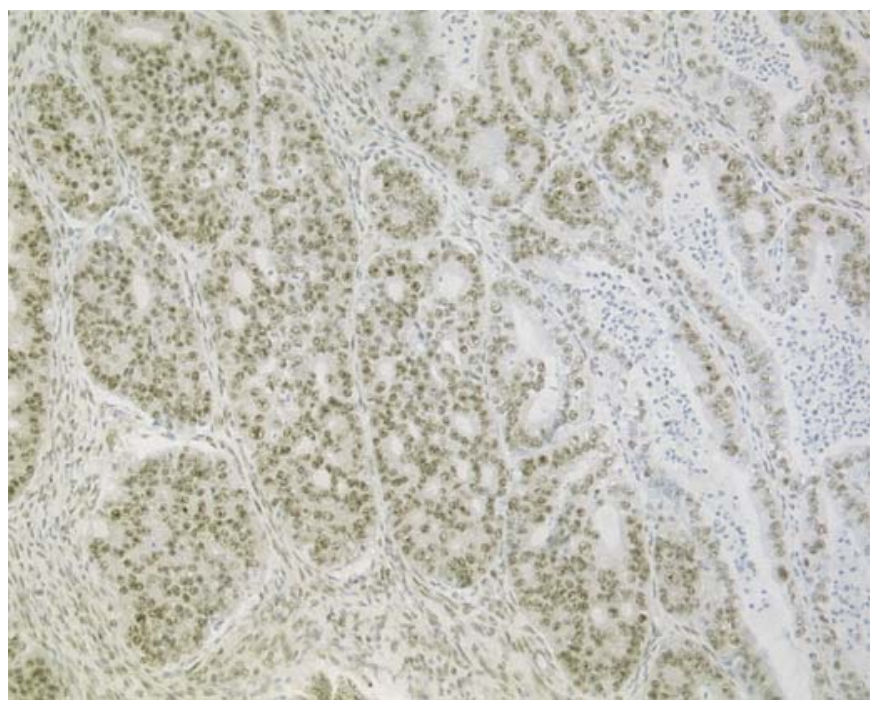

Figure 1. Histological distribution of glucocorticoid receptor expression in an invasive ovarian carcinoma. Nuclear signals are present in $>80 \%$ of tumor cells showing a moderate staining intensity (IRS 8, moderate expression). Original magnification, x10.

59.4; SD, 11.0). Median follow-up was 7.5 years (mean, 5.5 years; range, 4 days to 22.4 years).

Of the 85 tumors, $25(29.4 \%)$ were classed as FIGO stage I (growth limited to the ovaries), $23(27.1 \%)$ as stage II (growth involving one or both ovaries with pelvic extension), $29(34.1 \%)$ as stage III (tumor involving one or both ovaries with peritoneal implants outside the pelvis and/or positive retroperitoneal or inguinal nodes), and $8(9.4 \%)$ as stage IV (distant metastasis) (20).

Of 85 patients with stage $\mathrm{I} a / \mathrm{b}$ disease and a low tumor grading (grades 1 and 2), 4 had received no adjuvant treatment. Forty-four of 85 patients with disease stages between Ic and IIc had been treated with intraperitoneal chromic phosphate $\left(\mathrm{P}^{32}\right)$ and/or systemic chemotherapy according to the PEC regimen (cisplatinum $50 \mathrm{mg} / \mathrm{m}^{2}$, epirubicin $60 \mathrm{mg} / \mathrm{m}^{2}$ and cyclophosphamide $500 \mathrm{mg} / \mathrm{m}^{2}$; q28). Patients of all other stages received PEC chemotherapy, of which $69.4 \%$ also received radiotherapy, mainly via open field technique.

The success of postoperative therapy in patients with tumor residuals after surgery was assessed by second-look interventions, computed tomograms, radiography and sonography, and the course of CA125 serum values, as described previously (21).

Histological tumor classification. Of 85 invasive tumors, 48 $(56.5 \%)$ were classified as serous, $9(10.6 \%)$ as mucinous, 11 $(12.9 \%)$ as endometrioid, $12(14.1 \%)$ as clear cell, and 5 $(5.9 \%)$ as undifferentiated.

Glucocorticoid receptor immunohistochemistry. In general, the immunohistochemical staining of GR resulted in a clearly analyzable nuclear expression pattern of the receptor, which was similar to that of estrogen and progesterone receptors and allowed a simple discrimination of tumor cells and tumors (Fig. 1). Neither the staining intensity nor the percentage of positive tumor cells was found to depend on the age or storage time of the paraffin-embedded tumor specimens. 


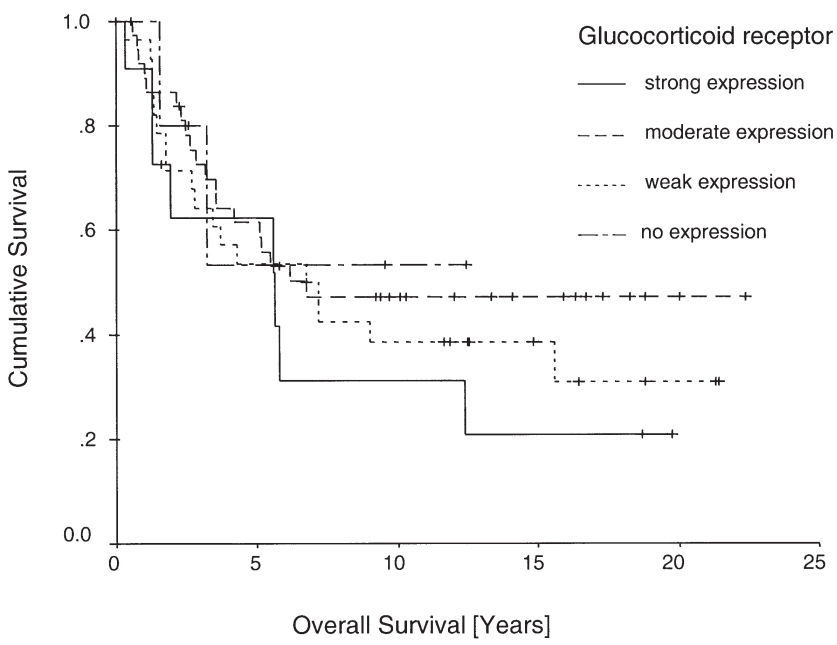

Figure 2. Kaplan-Meier survival analysis of glucocorticoid receptor expression.

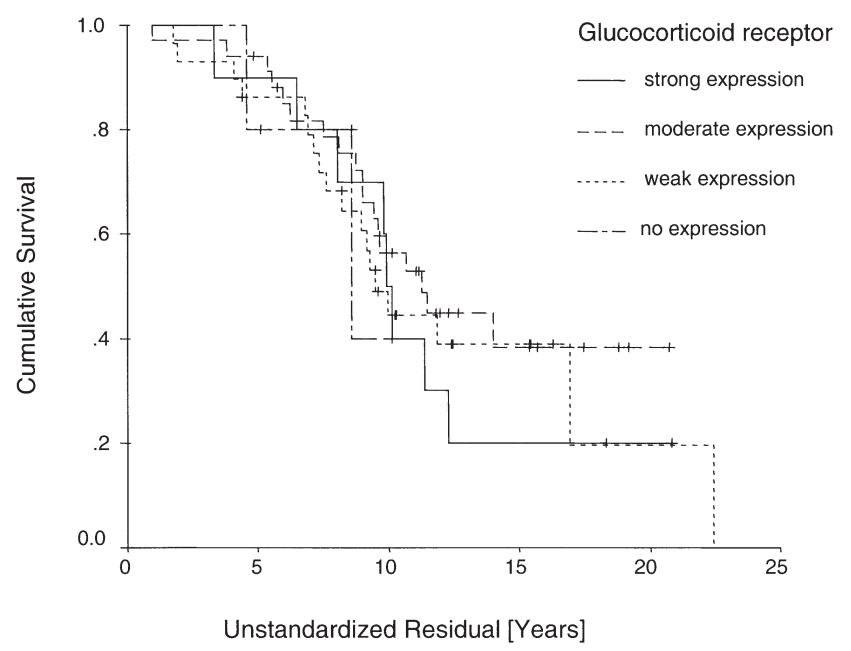

Figure 3. Kaplan-Meier residual survival analysis of glucocorticoid receptor expression.

Glucocorticoid receptor correlations and survival. Glucocorticoid receptor expression correlated with various clinical (age, disease stage), histopathological (grading and tumor type), and previously determined immunohistochemical factors (estrogen and progesterone receptors). No significant correlation or trend was observed. Thus, the antibody used does not cross-react with the steroid hormone receptors, estrogen and progesterone. Kaplan-Meier survival analysis showed that GR expression had no influence on overall survival (log-rank $=1.93 ; \mathrm{df}=3 ; \mathrm{p}=0.587 ;$ Fig. 2 ). Even residual Kaplan-Meier survival analysis, which included factors such as disease stage, tumor grading, success of initial therapy and age at diagnosis, showed no influence by GRs on overall survival (log-rank $0.93 ; \mathrm{df}=3 ; \mathrm{p}=0.819 ;$ Fig. 3 ).

Glucocorticoid receptor expression and administration. Sixteen patients received GCs as a part of antiemetic therapy ( 8 or $20 \mathrm{mg}$ of dexamethasone on day 1). The two subgroup analyses on GR expression and GC administration (yes versus no) showed no significant influence on survival $(\log -\mathrm{rank}=1.52 ; \mathrm{df}=3 ; \mathrm{p}=0.677)$.

\section{Discussion}

We were motivated to undertake this study because, despite the controversy, there are few clinical data on GC treatment or on the implications of GRs in solid tumors. This makes it difficult for clinicians to make balanced decisions about GC treatment.

Our results in ovarian cancer show that the degree of GR expression does not influence patient prognosis. Moreover, we found no evidence that $\mathrm{GC}$ treatment has an unfavorable effect on outcome, even when the tumor expresses the receptor. Although these findings may not allow us to draw final conclusions, it is important to realize that, in cases of GR expression, the receptors would also have been stimulated by endogenous cortisol during the circadian rhythm. We found no effects by GC treatment on the outcome of ovarian cancer patients.

With regard to the expression of GRs in ovarian cancer, there are no directly comparable clinical studies. A study of 91 patients with colon cancer also failed to find any correlation between GR expression and histopathological characteristics, proliferative capacity of tumors or patient survival (22). In contrast, a study of patients with resectable hepatocellular carcinoma found that those with GR-positive tumors had lower survival rates than those with GR-negative tumors (23). This may indicate that GRs have different roles in different tumor entities.

Previously published studies based on in vitro analyses that calculated the potential adverse effects of GC in antiemetic prophylaxis and older autopsy reports may have discouraged clinicians from using GCs. As a result, some patients may not have profited from the known potential benefits of GCs.

It is recognized that the duration of treatment, dosage, and dosing regimen, as well as the choice of appropriate GC and mode of application, can affect the risk/benefit ratio (24). These factors combined with individual susceptibility determine the occurrence and severity of adverse effects in all clinical situations, including the treatment of cancer (24). Glucocorticoids prescribed for antiemetic treatment are given for a short time only, the results of which may contrast the adverse effects reported in cases of long-term use. Analyses from the field of psychoneuroimmunology have shown that the short-term use of GC has cognitive benefits and long-term treatment is associated with negative effects on cognition (25).

In summary, the results of in vitro data alone provide insufficient evidence for making balanced decisions about $\mathrm{GC}$ treatment in cancer patients. To date, there are no clinical data indicating that GC should not be used in treating patients with ovarian cancer. We showed in this study that the expression of GR in ovarian cancer had no negative prognostic effects. In fact, the short-term use of GC may have additional hematoprotective effects in these patients (13).

\section{References}

1. Iversen HG and Hjort GH: The influence of corticoid steroids on the frequency of spleen metastases in patients with breast cancer. Acta Pathol Microbiol Scand 44: 205-212, 1958.

2. Sherlock P and Hartmann WH: Adrenal steroids and the pattern of metastases of breast cancer. J Am Med Assoc 181: 313-317, 1962. 
3. Haid M: Steroid antiemesis may be harmful. N Engl J Med 304: 1237,1981

4. Herr I, Ucur E, Herzer K, et al: Glucocorticoid cotreatment induces apoptosis resistance toward cancer therapy in carcinomas. Cancer Res 63: 3112-3120, 2003.

5. Runnebaum IB and Brüning A: Glucocorticoids inhibit cell death in ovarian cancer and up-regulate caspase inhibitor cIAP2. Clin Cancer Res 11: 6325-6332, 2005.

6. Rutz HP and Herr I: Interference of glucocorticoids with apoptosis signaling and host-tumor interactions. Cancer Biol Ther 3: 715-718, 2004.

7. Wu W, Chaudhuri S, Brickley DR, Pang D, Karrison T and Conzen SD: Microarray analysis reveals glucocorticoid-regulated survival genes that are associated with inhibition of apoptosis in breast epithelial cells. Cancer Res 64: 1757-1764, 2004

8. Braunschweiger PG, Ting HL and Schiffer LM: Correlation between glucocorticoid receptor content and the antiproliferative effect of dexamethasone in experimental solid tumors. Cancer Res 43: 4757-4761, 1983.

9. Iwai A, Fujii Y, Kawakami S, et al: Down-regulation of vascular endothelial growth factor in renal cell carcinoma cells by glucocorticoids. Mol Cell Endocrinol 226: 11-17, 2004.

10. Glick RD, Medary I, Aronson DC, Scotto KW, Swendeman SL and La Quaglia MP: The effects of serum depletion and dexamethasone on growth and differentiation of human neuroblastoma cell lines. J Pediatr Surg 35: 465-472, 2000.

11. Tissing WJ, Lauten M, Meijerink JP, et al: Expression of the glucocorticoid receptor and its isoforms in relation to glucocorticoid resistance in childhood acute lymphocytic leukemia. Haematologica 90: 1279-1281, 2005.

12. Koga Y, Matsuzaki A, Suminoe A, Hattori H, Kanemitsu S and Hara T: Differential mRNA expression of glucocorticoid receptor alpha and beta is associated with glucocorticoid sensitivity of acute lymphoblastic leukemia in children. Pediatr Blood Cancer 45: 121-127, 2005.

13. Munstedt K, Borces D, Bohlmann MK, Zygmunt M and von Georgi R: Glucocorticoid administration in antiemetic therapy: is it safe? Cancer 101: 1696-1702, 2004.
14. Piccart MJ, Bertelsen K, Stuart G, et al: Long-term follow-up confirms a survival advantage of the paclitaxel-cisplatin regimen over the cyclophosphamide-cisplatin combination in advanced ovarian cancer. Int J Gynecol Cancer 13: 144-148, 2003.

15. International Collaborative Ovarian Neoplasm Group: Paclitaxel plus carboplatin versus standard chemotherapy with either single-agent carboplatin or cyclophosphamide, doxorubicin, and cisplatin in women with ovarian cancer: the ICON3 randomised trial. Lancet 360: 505-515, 2002.

16. Russell P and Farnsworth A: Surgical pathology of the ovaries. 2nd edition. Churchill Livingston, London, 1997.

17. Baak JPA, Chan KK, Stolk JG and Kenemans P: Prognostic factors in borderline and invasive ovarian tumours of the common epithelial type. Path Res Pract 182: 755-774, 1987.

18. Münstedt K, Steen J, Knauf AG, Buch T, von Georgi R and Franke FE: Steroid hormone receptors and long term survival in invasive ovarian cancer. Cancer 89: 1783-1791, 2000.

19. Remmele W and Stegner HE: Vorschlag zur einheitlichen Definition eines Immunreaktiven Score (IRS) für den immunhistochemischen Östrogenrezeptor-Nachweis (ER-ICA) im Mammakarzinomgewebe. Pathologe 8: 138-140, 1987.

20. Wittekind CH, Meyer H-J and Bootz F: TNM, Klassifikation maligner Tumoren. 6th edition. Springer-Verlag, Berlin, 2002.

21. Von Georgi R, Schubert K, Grant P and Münstedt K: Posttherapy surveillance and after-care in ovarian cancer. Eur J Obstet Gynecol Reprod Biol 111: 189-196, 2003.

22. Theocharis S, Kouraklis G, Margeli A, et al: Glucocorticoid receptor (GR) immunohistochemical expression is correlated with cell cycle-related molecules in human colon cancer. Dig Dis Sci 48: 1745-1750, 2003

23. Ho WL, Wu CC, Yeh DC, et al: Roles of the glucocorticoid receptor in resectable hepatocellular carcinoma. Surgery 131: $19-25,2002$

24. Goodwin JS: Anti-inflammatory drugs. In: Basic and Clinical Immunology. Stities DP, Terr AI and Parslow TG (eds). 8th edition. Appleton and Lange, East Norwalk, pp786-795, 1994.

25. Lupien SJ and McEwen BS: The acute effects of corticosteroids on cognition: integration of animal and human model studies. Brain Res Brain Res Rev 24: 1-27, 1997. 